\title{
Modelos Matemáticos para Previsão de Resposta ao Tratamento com Hormônio de Crescimento
}

\begin{abstract}
RESUMO
Modelos preditivos de crescimento são algoritmos derivados de análises de regressão linear múltipla contendo variáveis que influenciam o crescimento em resposta ao tratamento com $\mathrm{GH}$ em determinado grupo de indivíduos durante determinado período. A partir do conhecimento da importância relativa de cada variável, são geradas fórmulas matemáticas que permitem obter uma medida objetiva do potencial de crescimento de cada indivíduo em resposta ao tratamento com $\mathrm{GH}$ em diferentes situações. Tais algoritmos podem, portanto, ser utilizados como ferramentas para a decisão fundamentada em evidência das estratégias de tratamento com $\mathrm{GH}$ a serem utilizadas em diferentes crianças com baixa estatura, a fim de se obter o máximo de custoefetividade com a menor dose cumulativa possível. Já foram desenvolvidos diversos modelos de previsão de resposta ao tratamento com GH em diferentes causas de baixa estatura, porém estes ainda apresentam baixa aplicabilidade clínica por causa do baixo poder preditivo e da baixa precisão da previsão, o que tem gerado crescente interesse pela incorporação de variáveis adicionais, como marcadores bioquímicos e genéticos que possam melhorar a acurácia da previsão e, assim, permitir que, no futuro, o tratamento com GH possa ser individualizado conforme as necessidades específicas de cada criança. (Arq Bras Endocrinol Metab 2008; 52/5:839-849)

Descritores: Hormônio do crescimento/deficiência; Hormônio do crescimento/farmacologia; Hormônio do crescimento/uso terapêutico; Farmacogenética; Nanismo; Insuficiência de crescimento
\end{abstract}

\begin{abstract}
Mathematical Models for Predicting Growth Responses to Growth Hormone Replacement Therapy.

Growth prediction models are algorithms derived from multiple regression analyses including variables that influence growth responses to $\mathrm{GH}$ therapy in a defined group of subjects over a defined period of time. Mathematical equations can be derived from the knowledge acquired with the relative importance of each variable, which provide objective measurements of each subject's growth potential in response to GH therapy on different situations. Therefore, these equations can be used as tools to improve evidence-based decision regarding to growth promoting treatment strategies to be used in each child, optimizing cost-effectiveness with the lowest cumulative GH dose. Several models have already been developed to predict growth responses to $\mathrm{GH}$ for different short stature causes, but they still have low clinical usefulness, due to their low predictive power and low prevision accuracy. This has lead to a growing interest in the addition of new variables, such as biochemical or genetic markers, which could improve prevision accuracy and then allow, in the future, $\mathrm{GH}$ therapy individualization according to the specific needs of each child. (Arq Bras Endocrinol Metab 2008; 52/5:839-849)
\end{abstract}

Keywords: Growth hormone/deficiency; Growth hormone/pharmacology; Growth hormone/therapeutic use; Pharmacogenetics; Dwarfism; Failure to thrive revisão

EVERLAyny F. Costalonga

Alexander A. L. Jorge

BERENICE B. MENDONÇA

IVO J. P. ARNHOLD

Unidade de Endocrinologia do

Desenvolvimento e Laboratório

de Hormônios e Genética

Molecular LIM/42, Disciplina de

Endocrinologia e Metabologia

do Hospital das Clínicas da

Faculdade de Medicina da

Universidade de São Paulo

(FMUSP), SP, Brasil.
Recebido em 17/4/2008

Aceito em 21/4/2008 


\section{INTRODUÇÃO}

$\mathrm{N}$ A DÉCADA DE 1950, o hormônio do crescimento growth hormone $(\mathrm{GH})$, obtido de hipófise de cadáver, foi pela primeira vez utilizado para tratar a baixa estatura de crianças com deficiência de GH (DGH). Na década de 1980, com o advento do hormônio de crescimento recombinante humano, houve crescente aumento do uso deste hormônio para pacientes com DGH, assim como com outras causas de baixa estatura. Hoje, cerca de 100 mil crianças são tratadas com GH em todo o mundo, mas a dose utilizada permanece largamente empírica. Nos Estados Unidos, por exemplo, doses diárias entre 0,025 e $0,050 \mathrm{mg} / \mathrm{kg} /$ dia são comumente usadas, enquanto na Europa as doses variam de 0,025 a $0,035 \mathrm{mg} / \mathrm{kg} /$ dia e no Japão não passam de 0,025 $\mathrm{mg} / \mathrm{kg} /$ dia (1). Ou seja, apesar de a extensa experiência de 40 anos de uso de GH para o tratamento de diferentes causas de baixa estatura, ainda não existe consenso quanto ao regime de tratamento ideal, e, dessa forma, a estratégia terapêutica se mantém inalterada, fundamentada em doses fixas, arbitrárias e ajustadas somente pelo peso, sem levar em consideração os diferentes graus de sensibilidade individual ao hormônio. Como resultado, o que se observa é ampla variabilidade de resposta ao tratamento, mesmo quando se comparam crianças da mesma categoria diagnóstica e tratadas segundo as mesmas recomendações (Figura 1).

Em virtude dessas observações, a necessidade de individualização do tratamento com GH conforme a sensibilidade e as características específicas de cada criança tem sido crescentemente reconhecida. Como passo inicial, rumo à individualização, inúmeros traba-
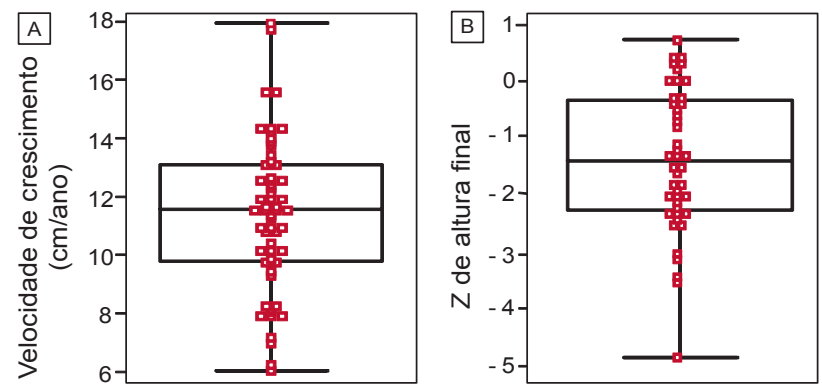

Figura 1. Variabilidade de resposta ao tratamento com $\mathrm{GH}$ em crianças com DGH. A) Velocidade de crescimento no primeiro ano de tratamento com $\mathrm{GH}$ de crianças pré-púberes com DGH; B) Z de altura final de crianças com DGH tratadas com $\mathrm{GH}$ por, pelo menos, três anos. lhos têm se dedicado ao estudo dos fatores determinantes da capacidade de resposta ao tratamento com $\mathrm{GH}$ nas diferentes causas de baixa estatura. A partir do conhecimento dessas variáveis e da importância relativa de cada uma, pode-se prever a resposta de crescimento que será obtida consoante a dose de $\mathrm{GH}$ utilizada em determinado indivíduo. De acordo com a previsão, o tratamento pode ser otimizado, a fim de se obter o máximo de crescimento com o mínimo de custos e riscos. Foi com esse objetivo que surgiram os chamados modelos preditivos de resposta ao tratamento com GH, que serão abordados nessa revisão, buscando proporcionar uma visão geral sobre os avanços até o momento no conhecimento dos fatores determinantes de resposta ao tratamento com GH (sobretudo na DGH) e os desafios a serem superados nos próximos anos para que essa modalidade terapêutica possa beneficiar, de maneira efetiva e segura, um número crescente de indivíduos.

\section{DEFINIÇÃO DE MODELOS PREDITIVOS}

Modelos preditivos de resposta ao tratamento com GH são algoritmos derivados de análises de regressão linear múltipla contendo variáveis que influenciam o crescimento em resposta ao tratamento com GH em determinado grupo de indivíduos durante um período definido (2). A partir do conhecimento da importância relativa de cada variável, são geradas fórmulas matemáticas que permitam obter a medida objetiva do potencial de crescimento de cada indivíduo em resposta ao tratamento com GH em diferentes situações.

\section{APLICABILIDADE CLÍNICA}

Os modelos preditivos de crescimento em resposta ao GH podem ser usados para:

- Melhor individualização do tratamento, ou seja, ajustar o tratamento conforme a sensibilidade individual, determinando assim a dose de GH ideal para se atingir a estatura-alvo de determinada criança de maneira rápida, efetiva e segura $(2,3)$.

- Permitir o desenvolvimento de estratégias de tratamento que utilizem a menor dose cumulativa possível, usando doses maiores nos momentos mais indicados pelos modelos preditivos (2) (para exemplo prático dessa aplicabilidade, consultar o site www.growthpredictions.org). 
- Minimizar possíveis riscos do tratamento.

- Reduzir custos financeiros.

- Identificar, desde o início, os possíveis "bons e maus respondedores", dessa forma auxiliando na tomada de decisão quanto ao início do tratamento.

- Propiciar aos pacientes, pais e agentes de saúde expectativas mais realistas de resposta do início ao final do tratamento $(2,3)$, a fim de prover base segura para encorajar ou desencorajar a continuação da terapia, fundamentando-se numa previsão acurada e não em um "simples desejo" do indivíduo.

- Monitorizar o tratamento passo a passo, de maneira que a observação de discrepâncias entre a resposta observada e a prevista ou de reduções inesperadas da resposta possam permitir a detecção precoce da presença de outros fatores interferentes, como falta de aderência ou doença endócrina ou não-endócrina subjacente não diagnosticadas $(2,3)$.

\section{CARACTERÍSTICAS DE UM MODELO PREDITIVO IDEAL}

Um modelo preditivo ideal deve ser confiável, eficiente e prático, a fim de que possa ser utilizado na prática clínica diária.

Objetivando confiabilidade dos resultados, o modelo preditivo deve ser desenvolvido a partir de dados de uma população ampla e representativa de uma categoria diagnóstica específica (DGH, síndrome de Turner (ST), baixa estatura idiopática etc.), com critérios de inclusão bem definidos que permitam a seleção da casuística o mais homogênea possível para que possa representar de maneira adequada os fatores determinantes de resposta naquele grupo específico.

Quanto às propriedades que, em última análise, determinam a aplicabilidade clínica do algoritmo para predição da resposta individual ao tratamento incluem:

- Alto poder preditivo $\left(\mathrm{R}^{2}\right)$ - significa ser capaz de explicar o grande percentual da variação de resposta que ocorre em determinada população (por exemplo, a previsão com $\mathrm{R}^{2}$ de 0,9 é capaz de explicar $90 \%$ da variabilidade que ocorre naquele grupo).

- Alta precisão da previsão - significa prever com baixo desvio-padrão (por exemplo, prever a velocidade de crescimento (VC) de $11 \mathrm{~cm} /$ ano com desvio-padrão de $0,4 \mathrm{~cm}$ significa prever que a $\mathrm{VC}$ será entre 10,2 e 11,8 (média \pm 2 DP) com 95\% de confiança).

- Utilizar para a previsão dados que estejam disponíveis já no início do tratamento, para que possam orientar a dose a ser utilizada desde a introdução do GH.

- Incluir dose de GH como variável, o que é essencial para que a resposta ao tratamento seja ajustada a partir da adequação deste fator (3).

- Ser "período específico" - como as condições que afetam o crescimento diferem conforme a idade, a puberdade e o tempo de tratamento, um bom modelo preditivo deve corresponder a séries de algoritmos descrevendo o crescimento em períodos seqüenciais, tipicamente para cada ano de tratamento ou, pelo menos, diferentes para os períodos pré e pós-puberais (3).

- Ser validado numa população independente e calibrado para valores extremos (2).

- Ser prático, simples, objetivo e integrado a um programa de informática de fácil utilização $(2,3)$.

- Ser regularmente atualizado para refletir mudanças das características clínicas e práticas de tratamento com GH (2).

\section{VALIDAÇÃO E CALIBRAÇÃO}

É importante destacar que o poder preditivo descrito para os diferentes modelos preditivos refere-se à população a partir da qual foi gerado o algoritmo, sendo provavelmente diferente quando aplicado a um segundo grupo de indivíduos. Além disso, sabe-se que os modelos preditivos apresentam performance aquém da esperada quando aplicados de maneira individual, mesmo em indivíduos da mesma população (4). Análises estatísticas recentes demonstram que, em muitos casos, isso ocorre por causa do chamado overfitting, fenômeno comum quando tanto a seleção dos preditores quanto a estimativa dos coeficientes são guiados pelo mesmo banco de dados, resultando "supervalorização" desses parâmetros e, conseqüentemente, em previsões muito extremas (5).

Portanto, para a válida aplicação de um modelo preditivo a novos indivíduos, as previsões devem ser não só validadas em uma segunda população, mas também calibradas para reduzir o overfitting. Para isso, o modelo preditivo é aplicado a uma população diferente daquela que originou os dados para a construção do algoritmo, verificando-se: a) qual o nível de precisão da 
previsão quando aplicado a um grupo de indivíduos diferente daquele usado para a análise de regressão linear múltipla; b) qual a acurácia da previsão quando aplicada de modo individual; c) se a aplicação deste modelo pode ser feita da forma como foi determinado originalmente ou por meio de ajustes matemáticos específicos.

As diferenças entre o crescimento observado e o predito em cada indivíduo podem ser expressas na forma do studentized residual, representado pela fórmula a seguir:

$\begin{aligned} & \text { Studentized } \\ & \text { residual }\end{aligned}=\frac{\text { crescimento observado }- \text { predito }}{\text { Erro-padrão (SE) calculado para cada indivíduo }}$

Este é um cálculo comparável ao do escore de desvio-padrão ("Z"). Valores ao redor de zero indicam resposta compatível com a esperada pelo modelo preditivo (2). A partir deste cálculo podem ser construídas curvas de calibração que representam as diferenças entre o observado e o previsto em cada criança, indicando, dessa forma, o nível de acurácia do modelo preditivo quando aplicado a um novo grupo de indivíduos.

\section{CARACTERÍSTICAS GERAIS}

\section{Classificação}

Como comentado anteriormente, um modelo preditivo ideal deve ser diagnóstico e período-específico. Assim, os modelos preditivos podem ser classificados quanto a:

\section{Categoria diagnóstica}

Já existem modelos disponíveis para previsão da resposta ao tratamento com GH em DGH (5-10), ST (11-15), baixa estatura idiopática $(14,16,17)$ e nascidos pequenos para a idade gestacional (PIGs) $(18,19)$.

\section{Período do crescimento estudado}

Em pacientes com DGH, por exemplo, existem estudos avaliando os fatores preditivos especificamente em indivíduos menores de 3 anos de idade (20), em crianças de 1 a 8 anos (pré-púberes) (10) e durante o período puberal (21).

\section{Variáveis utilizadas para a previsão}

Em termos gerais, as variáveis analisadas na maior parte dos modelos desenvolvidos até o momento foram relacionadas a: a) nascimento - peso, altura, sexo; b) potencial genético - altura dos pais; c) início do tratamento - idade, altura, peso, estádio puberal; d) parâmetros laboratoriais - sobretudo pico máximo de GH em testes de estímulo; e) modalidade terapêutica - dose, freqüência das injeções, duração do tratamento.

\section{MODELOS PREDITIVOS EM CRIANÇAS COM DGH}

Os modelos de predição de resposta de crescimento ao tratamento com GH em crianças com DGH podem ser classificados de maneira simplificada em modelos de predição para a resposta a curto prazo (VC) e ganho em $\mathrm{Z}$ de altura no primeiro ano de tratamento) e a longo prazo $(Z$ de altura final, com e sem ajuste pelo alvo e ganho em $\mathrm{Z}$ de altura total ao longo do tratamento).

As principais variáveis que demonstraram influenciar a resposta ao tratamento com $\mathrm{GH}$ a curto e a longo prazo em crianças com DGH encontram-se resumidamente descritas na Tabela 1.

Tabela 1. Fatores determinantes de resposta ao tratamento com GH a curto e a longo prazos em crianças com DGH*.

\begin{tabular}{|c|c|}
\hline $\begin{array}{l}\text { Resposta a curto prazo } \\
\left.\text { ( } 1^{\circ} \text { ano }\right)\end{array}$ & $\begin{array}{c}\text { Resposta a longo prazo } \\
\text { (altura final) }\end{array}$ \\
\hline Pico de GH máximo em testes de estímulo $(10,20,22-24)$ & Resposta de crescimento no $1^{\circ}$ ano $(5,7,9,25)$ \\
\hline Idade cronológica no início do tratamento $(10,20,22-24)$ & Estatura-alvo $(5,8,9,25)$ \\
\hline $\begin{array}{l}Z \text { de altura inicial com }(10) \text { e sem }(23,24) \\
\text { ajuste para estatura-alvo }\end{array}$ & Pico de GH máximo em testes de estímulo $(5,8,9,25)$ \\
\hline Estatura-alvo $(20,22-24)$ & $Z$ de altura inicial $(5,7,8,25,26)$ \\
\hline Dose utilizada $(1,10,20,22,27-29)$ & Duração do tratamento $(7-9,25)$ \\
\hline Z de peso no início do tratamento $(10,20)$ & Idade cronológica no início do tratamento $(7,9)$ \\
\hline$Z$ de peso ao nascer $(10,20)$ & Sexo $(5,7,8)$ \\
\hline Número de injeções/semana $(23,31)$ & $\begin{array}{l}\text { Presença de deficiência hormonal múltipla }(5,25) \\
\text { Puberdade espontânea ou induzida }(17,25,26,30) \\
\text { Idade ou altura do início da puberdade }(6,8,32)\end{array}$ \\
\hline
\end{tabular}




\section{Modelos de previsão de resposta a curto prazo (primeiro ano de tratamento)}

Diversos estudos procuraram identificar os fatores preditivos de resposta ao tratamento com GH em crianças com DGH no primeiro ano de tratamento $(10,20,22-24)$.

Entre estes, destaca-se o trabalho desenvolvido por Ranke e cols. (10), 1999, envolvendo 593 crianças do banco de dados KIGS (Pfizer International Growth Study Database), exclusivamente pré-púberes, com DGH idiopática diagnosticada por pico máximo de GH $<10 \mathrm{ng} / \mathrm{ml} \mathrm{em} \mathrm{pelos} \mathrm{menos} \mathrm{dois} \mathrm{testes} \mathrm{de} \mathrm{estímulo} \mathrm{e}$ tratadas exclusivamente com $\mathrm{GH}$ recombinante na dose média de $0,6 \mathrm{UI} / \mathrm{kg} / \mathrm{semana}$. O estudo se destacou em relação aos trabalhos prévios em virtude da ampla casuística utilizada e por ter restringido a análise a pacientes recebendo pelo menos 6 injeções/semana e que tinham registros de $\mathrm{VC}$ aferida com 11 a 13 meses de tratamento.

A variável de maior importância para a VC no primeiro ano de tratamento foi o logaritmo natural da resposta máxima de $\mathrm{GH}$ em testes provocativos, a qual se demonstrou inversamente relacionada à VC. Ou seja, quanto maior a gravidade da $\mathrm{DGH}$, melhor a resposta ao tratamento no primeiro ano. Além disso, a VC no primeiro ano foi inversamente relacionada à idade cronológica e ao $\mathrm{Z}$ de altura no início do tratamento (ajustado para a estatura-alvo); e positivamente correlacionada ao $\mathrm{Z}$ peso inicial, logaritmo natural da dose semanal e $\mathrm{Z}$ de peso ao nascer. A contribuição relativa de cada um desses fatores identificada pela análise de regressão linear múltipla está ilustrada na Tabela 2.

Tabela 2. Modelo de regressão linear múltipla para predição da VC no primeiro ano de tratamento com $\mathrm{GH}$ em crianças com DGH (com base nos dados do KIGS, adaptado de Ranke e cols., JCEM, 1999) (10).

\begin{tabular}{lcc}
\hline Variável de predição & Coeficiente & Rank \\
Ln do pico $\mathrm{GH}$ máximo $(\mu \mathrm{g} / \mathrm{dl})$ & $-1,37$ & 1 \\
\hline Idade cronológica inicial & $-0,32$ & 2 \\
\hline $\begin{array}{l}\text { Z de altura inicial - Z da } \\
\text { midparental heigth }(\mathrm{MPH})\end{array}$ & $-0,4$ & 3 \\
Z de peso inicial & 0,29 & 4 \\
\hline Ln da dose (Ul/kg/semana) & 1,62 & 5 \\
\hline Z do peso ao nascer & 0,32 & 6 \\
Constante & \multicolumn{3}{c}{14,55} \\
R & \multicolumn{3}{c}{0,61} \\
DP & \multicolumn{3}{c}{1,46} \\
\hline L & & \\
\hline
\end{tabular}

$\mathrm{Ln}=\mathrm{Log}$ natural; $\mathrm{Z}$ da midparental height $(\mathrm{MPH})=(\mathrm{Z}$ de altura do pai $+\mathrm{Z}$ de altura da mãe) $\div$ 1,61; DP = desvio-padrão.
A equação que descreve a predição da VC primeiro ano a partir da análise mencionada é:

VC predita $=14,55+(-1,37 \times$ Ln GHmáx $)+(-0,32$ $\times \mathrm{IC}$ inicial $)+(0,32 \times \mathrm{Z}$ peso ao nascer $)+(1,62 \times \mathrm{Ln}$ dose $\mathrm{GH})+(-0,4 \times \mathrm{Z}$ altura inicial $-\mathrm{Z} \mathrm{MP})+(0,29$ $\times \mathrm{Z}$ peso inicial $)( \pm 1,46 \mathrm{~cm} / \mathrm{ano})$

Como descrito na Tabela 2, o modelo explicava $\left(\mathrm{R}^{2}\right)$ 61\% da variabilidade da VC no primeiro ano de tratamento com desvio-padrão de $1,46 \mathrm{~cm}$. De modo interessante, os autores construíram um segundo modelo em que o pico de GH máximo em testes de estímulo era excluído da equação; e observaram que o modelo resultante apresentava menor poder preditivo (45\%), maior desvio-padrão $(1,76 \mathrm{~cm})$ e tendência a "subprever" a resposta de crescimento das crianças com DGH mais grave (pico GH máx $<5 \mathrm{ng} / \mathrm{ml}$, sobretudo $<3 \mathrm{ng} / \mathrm{ml}$ ). Tal observação sugere que a importância do pico de GH como preditor de resposta seja ainda mais evidente quando se comparam indivíduos com pico de GH máximo muito baixo - provavelmente os "verdadeiros DGH" - e os portadores de "DGH parcial", provavelmente incluindo alguns pacientes sem deficiência real de GH. Assim, em razão de os primeiros apresentarem respostas de crescimento nitidamente superiores aos últimos, a tentativa de uso de um modelo comum aos dois grupos sem considerar o que os diferencia com certa segurança - que é o pico de GH em testes de estímulo - resulta previsões equivocadas no grupo com verdadeira DGH, quando comparadas aos casos de baixa estatura com etiopatogenia não claramente relacionada à $\mathrm{DGH}$.

\section{Modelos de previsão de resposta a longo prazo (altura final)}

Os trabalhos avaliando a resposta ao tratamento com $\mathrm{GH}$ em termos de altura final são mais numerosos e também mais heterogêneos, com alguns incluindo somente pacientes com DGH isolada $(6,7)$ e outros incluindo pacientes com deficiências hipofisárias múltiplas $(5,8,9,25)$, por exemplo.

Destaca-se o modelo desenvolvido por Ridder e cols. (5), 2007, que se diferencia dos demais pela primorosa análise estatística e pelo cuidado de construir modelos diferentes conforme a presença ou não da 
puberdade no primeiro ano de tratamento. Além disso, se propõe a construir um modelo preditivo do $\mathrm{Z}$ de altura final que possa ser aplicável no início do tratamento, ou seja, sem dados retrospectivos, como VC no primeiro ano e variáveis relacionadas à puberdade, os quais são utilizados na maioria dos modelos preditivos de altura final, dessa forma impedindo sua aplicabilidade clínica no início do tratamento.

$\mathrm{O}$ modelo envolvendo exclusivamente pacientes pré-púberes do início ao final do primeiro ano de tratamento e utilizando apenas dados disponíveis no início do tratamento pode ser descrito pela equação a seguir e pela Tabela 3:

$\mathbf{Z}$ de altura final $=1,186+1,021 \times \mathrm{Z}$ altura inicial + $0,077 \times(\mathrm{Z} \text { altura inicial })^{2}+0,264 \times \mathrm{Z}$ da estaturaalvo $-0,148 \times \operatorname{Ln}(\max G H)+0,260 \times \operatorname{sexo}+0,302$ $\times \mathrm{DHM}-0,047 \times$ idade óssea inicial $( \pm 0,84 \mathrm{DP})$

Este modelo foi capaz de prever $37 \%$ da variabilidade de resposta em termos de $\mathrm{Z}$ de altura final com desvio-padrão e $\pm 0,84 \mathrm{DP}$.

\section{MODELOS PREDITIVOS EM OUTRAS CAUSAS DE BAIXA ESTATURA}

Embora se tenha optado, por questões didáticas, por não entrar em detalhes sobre os modelos preditivos de resposta ao tratamento com $\mathrm{GH}$ nas baixas estaturas de etiologia diferente da DGH, é válida a análise comparativa breve dos fatores que se demonstraram importantes na determinação de resposta ao GH em diferentes causas de baixa estatura.

Enquanto na DGH o preditor mais importante foi o grau de DGH, nos indivíduos com ST ou nascidos pequenos para a idade gestacional, por exemplo, o fator mais importante foi a dose de $\mathrm{GH}$ utilizada, seguida pela idade cronológica do início do tratamento. A título de ilustração, a Tabela 4 faz uma análise comparativa do grau de importância de cada variável na predição da VC no primeiro ano de tratamento com $\mathrm{GH}$ em indivíduos com DGH, ST e PIGs, segundo os modelos construídos com base no banco de dados do KIGS. Observe que, a partir das variáveis analisadas, pode-se explicar $61 \%$ da variabilidade de resposta ao tratamento com $\mathrm{GH}$ em crianças com DGH, $46 \%$ em crianças com ST e $52 \%$ em crianças nascidas PIG. A maior capacidade preditiva em crianças com DGH já seria esperada, por se tratar de um grupo teoricamente mais homogêneo em termos de fisiopatologia e de tratamento da causa da baixa estatura em relação aos demais grupos diagnósticos.

\section{EXEMPLOS DE APLICAÇÃO DE MODELOS PREDITIVOS}

A título de ilustração, considere-se uma criança que no momento do diagnóstico de DGH apresente as seguin-

Tabela 3. Modelo de regressão linear múltipla para predição do Z de altura final após tratamento com GH em crianças com DGH, usando apenas dados disponíveis já no início do tratamento (adaptado de Ridder e cols., JCEM, 2007) (5).

\begin{tabular}{|c|c|c|c|}
\hline Variável de predição & Coeficiente & $p$ & $\mathbf{R}^{2}$ parcial \\
\hline Z de altura inicial & 1,902 & $<0,0001$ & 0,239 \\
\hline$(Z \text { de altura inicial })^{2}$ & 0,082 & 0,02 & \\
\hline Z da estatura-alvo & 0,282 & $<0,0001$ & 0,086 \\
\hline Ln do pico de GH máximo (ng/ml) & $-0,158$ & 0,02 & 0,022 \\
\hline $\operatorname{Sexo}(0=$ masc $. / 1=$ fem. $)$ & 0,278 & 0,01 & 0,022 \\
\hline Deficiência hipofisária múltipla (DHM) (0 = não/1 = sim) & 0,323 & 0,01 & 0,023 \\
\hline Idade óssea inicial (anos) & $-0,051$ & 0,003 & 0,031 \\
\hline Constante & 1,399 & 0,006 & \\
\hline $\mathbf{R}^{\mathbf{2}^{*}}$ & & & 0,37 \\
\hline DP & & & 0,84 \\
\hline
\end{tabular}

Ln = log natural; * corrigidos para o chamado overfitting (ajuste de valores extremos); DP = desvio-padrão. 
Tabela 4. Análise comparativa das variáveis determinantes da VC no primeiro ano de tratamento com GH em indivíduos com DGH, ST e nascidos PIGs, segundo modelos do KIGS.

\begin{tabular}{|c|c|c|c|c|c|c|}
\hline \multirow[t]{2}{*}{ Variáveis de predição } & \multicolumn{2}{|c|}{ DGH } & \multicolumn{2}{|c|}{ ST } & \multicolumn{2}{|c|}{ PIG } \\
\hline & Coeficiente & Rank & Coeficiente & Rank & Coeficiente & Rank \\
\hline Pico de GH máximo & $-1,37$ & 1 & - & - & - & - \\
\hline Dose de GH & 1,62 & 5 & 2,2 & 1 & 56,5 & 1 \\
\hline Idade inicial & $-0,32$ & 2 & $-0,3$ & 2 & $-0,3$ & 2 \\
\hline $\begin{array}{l}\text { Z de altura ajustado para o Z da } \\
\text { estatura-alvo }\end{array}$ & $-0,4$ & 3 & $-0,2$ & 5 & - & - \\
\hline Z da MPH & - & - & - & - & 0,11 & 4 \\
\hline Z de peso inicial & 0,29 & 4 & 0,4 & 3 & 0,3 & 3 \\
\hline Z de peso ao nascer & 0,32 & 6 & - & - & - & - \\
\hline $\mathrm{R}^{2}$ & \multicolumn{2}{|c|}{0,61} & \multicolumn{2}{|c|}{0,46} & \multicolumn{2}{|c|}{0,52} \\
\hline DP & \multicolumn{2}{|c|}{1,5} & \multicolumn{2}{|c|}{1,3} & \multicolumn{2}{|c|}{1,3} \\
\hline
\end{tabular}

$\mathrm{DP}=$ desvio-padrão.

tes características: sexo masculino; DGH isolada; idade cronológica $=6$ anos; idade óssea $=3$ anos; GH máximo obtido em testes de estímulo $=2,0(\mathrm{ng} / \mathrm{ml}) ; \mathrm{Z}$ da altura atual $=-4,2 \mathrm{DP} ; \mathrm{Z}$ de peso atual $=+1 \mathrm{DP} ; \mathrm{Z}$ da $\mathrm{MPH}=-0,15 \mathrm{DP} ; \mathrm{Z}$ da estatura-alvo $=-0,20 \mathrm{DP} ; \mathrm{Z}$ do peso ao nascer $=+0,2 \mathrm{DP}$.

Utilizando a dose de $\mathrm{GH}$ de $0,1 \mathrm{UI} / \mathrm{kg} / \mathrm{dia}(=0,7$ $\mathrm{UI} / \mathrm{kg} / \mathrm{semana}$ ), qual seria a VC do primeiro ano e a altura final esperadas?

Utilizando como modelo de previsão para a VC no primeiro ano de tratamento, a equação desenvolvida por Ranke e cols. (11), 1999, teríamos:

VC $1^{\circ}$ ano esperada $=14,55+(-1,37 \times \operatorname{Ln} 2)+(-0,32$ $\times 6)+(0,32 \times 0,2)+(1,62 \times \operatorname{Ln} 0,7)+\{-0,4 \times[(-4,2)$ $-(-0,15)]\}+(0,29 \times 11)=13,1 \pm 1,46 \mathrm{~cm} /$ ano

Já para a previsão do $\mathrm{Z}$ de altura final, se utilizar o modelo de Ridder e cols. (5), o qual se destaca por ser aplicável no início do tratamento utilizando apenas parâmetros disponíveis já no momento do diagnóstico:

$\mathbf{Z}$ de altura final esperado $=1.186+1,021 \times(-4,2)$ $+0,077 \times(-4,2)^{2}+0,264 \times(-0,2)-(0,148 \times \operatorname{Ln} 2)$ $+(0,260 \times 0)+(0,302 \times 0)-(0,047 \times 3)=-2,0 \pm$ $0,84 \mathrm{DP}$

\section{ANÁLISE CRÍTICA DOS MODELOS PREDITIVOS ATUAIS}

\section{Vieses de seleção da casuística para estudo}

Ao se analisar os resultados de estudos referentes ao tratamento com GH, é importante considerar que a maioria deles foi desenvolvida com base em grandes bancos de dados de registros nacionais e internacionais, estando sujeitos a inerente heterogeneidade da amostra (25). Além disso, os critérios diagnósticos utilizados na maior parte das vezes - pico de GH máximo em testes de estímulo $<10 \mathrm{ng} / \mathrm{ml}$ - são arbitrários, inespecíficos $(33,34)$, provavelmente inadequados para os ensaios monoclonais atuais (28), além de não necessariamente levarem em consideração os critérios clínicos e auxológicos sabidamente importantes para a definição do diagnóstico de DGH.

Sendo assim, diversos autores $(8,23,25,34-36)$ têm apontado evidências de que uma proporção considerável de indivíduos considerados como tendo DGH nesses estudos apresentem na verdade condições como baixa estatura idiopática ou retardo constitucional do crescimento e puberdade, o que prejudica a interpretação dos dados levantados e, conseqüentemente, a qualidade da previsão de resposta. Uma dessas evidências é a alta taxa de não-confirmação da DGH quando os indivíduos são retestados na idade adulta (37-39). Outra é a forte correlação observada entre o pico máximo de resposta do $\mathrm{GH}$ em testes de estímulo e a resposta ao tratamento com GH, sobretudo no primeiro ano de 
tratamento. Afinal, é indiscutível que pacientes com DGH verdadeira apresentam resposta ao tratamento com GH obviamente superior à de crianças com baixa estatura idiopática e DGH "questionável" (35); e que o pico máximo de $\mathrm{GH}$ é um dos parâmetros que mais bem refletem a presença ou não de $\mathrm{DGH}$ verdadeira nesses indivíduos $(8,17,35,36)$.

\section{Baixo poder preditivo e baixa precisão da previsão}

Note-se que há diferença entre conhecer os fatores determinantes de resposta ao tratamento com GH e desenvolver um modelo preditivo que possa ser utilizado na prática clínica para o ajuste da estratégia terapêutica. Apesar de já terem sido identificados diversos fatores determinantes de resposta ao tratamento com GH, a aplicabilidade clínica deste conhecimento ainda é limitada pelo baixo poder preditivo e o amplo desvio-padrão com que a previsão é feita (Tabela 5), o que desencoraja sua utilização na prática diária.

$\mathrm{O}$ baixo poder preditivo $\left(\mathrm{R}^{2}\right)$ observado sugere que fatores determinantes importantes ainda estejam por ser identificados; e dessa forma tem sido crescente o número de trabalhos buscando identificar outras variáveis, como parâmetros bioquímicos [IGFl, IGFBP3, marcadores de remodelação óssea (24)] e marcadores genéticos que possam refletir, de modo adicional, a sensibilidade ao tratamento com GH.

Além disso, não basta que o modelo tenha boa capacidade preditiva para que seja aplicável clinicamente: se o desvio-padrão for alto (baixa precisão), o algoritmo representa essencialmente a "descrição" das variáveis que se correlacionam à resposta ao tratamento $\mathrm{e}$ não permite sua utilização na prática clínica de maneira individual. No exemplo descrito anteriormente, podemos afirmar que o $\mathrm{Z}$ de altura final daquela criança ficaria entre $-3,72$ e $-0,36$ (previsão $\pm 2 \mathrm{DP}$ ) com $95 \%$ de confiança. Tal imprecisão da previsão inviabiliza a pretendida "tomada de decisão com base em evidências" pelos modelos preditivos, evidenciando, mais uma vez, a necessidade de melhora da acurácia do método.

\section{Ausência de estudos prospectivos}

É importante ressaltar que todos os estudos disponíveis até o momento sobre os resultados da terapia com GH em diferentes causas de baixa estatura e as tentativas de construção de modelos preditivos são fundamentados em dados retrospectivos/observacionais, o que impede qualquer conclusão definitiva.

Assim, embora a idéia de individualização da dose de $\mathrm{GH}$, desde o início do tratamento a partir do uso de modelos preditivos, pareça uma estratégia adequada para se atingir o objetivo de proporcionar o máximo de benefício com o uso da menor dose cumulativa possível de $\mathrm{GH}$, esta hipótese ainda não foi testada em estudos prospectivos e controlados.

\section{PERSPECTIVAS: A FARMACOGENÔMICA DO TRATAMENTO COM GH}

A baixa capacidade preditiva e o amplo desvio-padrão da previsão feita com base nas variáveis conhecidas até o momento sugerem que importantes fatores determinantes de sensibilidade ao tratamento com GH ainda estejam por ser reconhecidos. Considerando-se que $75 \%$ a

Tabela 5. Poder preditivo e desvio-padrão para alguns dos principais modelos preditivos de resposta ao tratamento com GH em crianças com DGH.

\begin{tabular}{|c|c|c|c|}
\hline & Autor/revista/ano & Poder preditivo & Desvio-padrão \\
\hline \multirow[t]{3}{*}{$\operatorname{VC} 1^{\circ}$ ano } & Blethen e cols., JCEM, 1993 (22) & $40 \%$ & ** \\
\hline & Ranke e cols., JCEM, 1999 (3) & $61 \%$ & $1,46 \mathrm{~cm} / \mathrm{ano}$ \\
\hline & Cole e cols., Arch Dis Child, 2004 (23) & $42 \%$ & $0,29 \mathrm{DP}$ \\
\hline \multirow[t]{4}{*}{ Altura final } & Blethen e cols., JCEM, 1997 (7) & $47 \%$ & ** \\
\hline & Carel e cols., BMJ, 2002 (8) & $58 \%$ & ** \\
\hline & Reiter e cols., JCEM, 2006 (25) & $56 \%$ & ** \\
\hline & Ridder e cols., JCEM, 2007* (5) & $37 \%$ & $0,84 \mathrm{DP}$ \\
\hline
\end{tabular}

* Modelo incluindo apenas dados disponíveis no início do tratamento e aplicável para crianças pré-púberes do início ao final do primeiro ano de tratamento; ** Dados não fornecidos nos artigos. 
90\% da altura de um indivíduo normal seja determinada geneticamente, e que a estatura-alvo foi um importante fator preditivo em diferentes modelos $(5,8,9,25)$, é bastante provável que fatores genéticos sejam fundamentais na determinação dessa resposta. Assim, tem sido crescente na literatura o interesse pelo estudo de variantes genéticas que possam influenciar a resposta ao tratamento com GH - a chamada "farmacogenômica do tratamento com hormônio de crescimento".

Os primeiros trabalhos nessa área tiveram como objeto de estudo o gene do receptor do GH (GHR), já que este é a primeira molécula-chave envolvida na mediação das ações do GH. Na população geral, existem duas isoformas do GHR: uma completa (GHRfl full-lenght) e uma apresentando deleção do éxon 3 (GHRd3), presente em cerca de 50\% dos indivíduos normais. Embora as duas isoformas apresentem afinidades semelhantes pelo GH, estudos funcionais demonstraram que células transientemente transfectadas com GHRd3 induzem maior transdução do sinal após tratamento com GH do que células transfectadas com o GHRfl. Em 2004, Santos e cols. (40) demonstraram que indivíduos apresentando pelo menos um alelo deletado para o exon 3 apresentavam VC no primeiro e no segundo ano de tratamento com GH superior à de indivíduos apresentando apenas o receptor full-lenght. Em 2006, nosso grupo demonstrou a influência positiva desse mesmo polimorfismo sobre a resposta ao tratamento com GH de crianças com DGH grave, tanto em relação à VC no primeiro ano quanto à altura final (4l). Embora análises subseqüentes não tenham sido uniformes quanto a esta observação, estes estudos introduziram o importante conceito de que variações comuns em genes envolvidos no eixo GH - IGFl podem desempenhar papel na predição de resposta à terapia com $\mathrm{GH}(41)$.

É provável que nos próximos anos sejam identificados marcadores adicionais que permitam melhor caracterização da base genética envolvida com a resposta ao tratamento com GH e possivelmente melhora da acurácia de previsão.

\section{CONCLUSÕES}

A extensa experiência obtida até o momento com o uso de $\mathrm{GH}$, em doses fixas com base no peso, torna evidente a necessidade de individualização das estratégias terapêuticas, a fim de que os objetivos do tratamento sejam alcançados de maneira mais homogênea entre os indivíduos. Hoje, sabe-se que diversos aspectos relacionados ao perfil biológico de cada indivíduo podem afetar a forma como este responde a dado regime terapêutico, e que por isso indivíduos com o mesmo diagnóstico e tratados segundo as mesmas recomendações apresentam respostas de crescimento tão variáveis. Fatores como a idade cronológica e o grau de déficit de altura observados no início do tratamento, a estatura-alvo e a dose de GH utilizada já estão bem estabelecidos como determinantes de resposta ao tratamento com GH. Além disso, sabe-se que determinada dose cumulativa de GH pode apresentar diferentes resultados finais, dependendo da forma como a dose é fracionada ao longo do tratamento.

A terapia individualizada fundamenta-se no ajuste da estratégia terapêutica conforme o perfil de sensibilidade de cada indivíduo, a fim de se obter o máximo de benefício com a mínima dose cumulativa possível. Idealmente, a terapia individualizada deve ser aplicada desde o momento da decisão quanto ao uso ou não do $\mathrm{GH}$ e com base em ajustes periódicos nas diferentes etapas do desenvolvimento da criança. Para que isso seja possível, no entanto, são necessárias ferramentas que permitam prever de maneira acurada e objetiva o potencial de resposta de cada indivíduo.

Nesse sentido, o desenvolvimento de modelos preditivos específicos para cada categoria diagnóstica e cada etapa do desenvolvimento constitui importante passo rumo à modernização e à otimização da terapia com GH. Algoritmos adequadamente precisos e que incluam a dose de $\mathrm{GH}$ como variável podem ser usados para prever a resposta de crescimento que será obtida com o uso de determinada dose de GH durante determinado período e, a partir disso, orientar o planejamento da estratégia terapêutica a ser utilizada durante o curso do tratamento. Além disso, permitem expectativas mais realistas quanto aos resultados, auxiliando, assim, de modo objetivo, a tomada de decisão quanto ao início do tratamento.

Com base nos conhecimentos obtidos até o momento, é possível prever cerca de $50 \%$ da variabilidade de resposta ao tratamento com GH. Essa baixa capacidade preditiva indica que fatores determinantes genéticos e/ou ambientais ainda estejam por ser reconhecidos. Os resultados iniciais dos estudos de farmacogenômica do tratamento com $\mathrm{GH}$, indicando que um polimorfismo do GHR possa estar relacionado à capacidade de resposta ao tratamento com GH, sugerem que, no futuro, es- 
tudos de farmacogenética possam prover ferramentas adicionais para o refinamento dos modelos preditivos, permitindo, dessa forma, maior capacidade preditiva e, conseqüentemente, melhor precisão da previsão.

Embora estudos prospectivos sejam necessários para confirmar esta afirmação, é provável que a partir de instrumentos de previsão mais acurados possamos, finalmente, transferir a individualização do tratamento com GH do campo da teoria para a prática, assim, otimizando o custo-efetividade do tratamento e suplantando a tradicional abordagem terapêutica fundamentada no peso que vem sendo utilizada nos últimos 40 anos de experiência com $\mathrm{GH}$.

\section{AGRADECIMENTOS}

Este trabalho obteve apoio da Fundação de Amparo à Pesquisa do Estado de São Paulo (Fapesp): Projeto Temático no $05 / 04726-0$ e bolsa no $06 / 55763-5$ para EFC; e do Conselho Nacional de Desenvolvimento Científico e Tecnológico (CNPq): 307951/06-5 para AALJ, 301246/95-5 para BBM e 300938/06-3 para IJPA.

\section{REFERÊNCIAS}

1. Cohen P, Bright GM, Rogol AD, Kappelgaard AM, Rosenfeld RG. Effects of dose and gender on the growth and growth factor response to $\mathrm{GH}$ in $\mathrm{GH}$-deficient children: implications for efficacy and safety. J Clin Endocrinol Metab. 2002;87:90-8.

2. Geffner ME, Dunger DB. Future directions: growth prediction models. Horm Res. 2007;68 Suppl 5:51-6.

3. Ranke MB. Prediction of response to GH: does it help to individualize therapy? Curr Opin Endocrinol Diabetes. 1999;6:93-9.

4. Ridder MA, Stijnen T, Hokken-Koelega AC. Validation and calibration of the Kabi Pharmacia International Growth Study prediction model for children with idiopathic growth hormone deficiency. J Clin Endocrinol Metab. 2003;88:1223-27.

5. Ridder MA, Stijnen T, Hokken-Koelega AC. Prediction of adult height in growth-hormone-treated children with growth hormone deficiency. J Clin Endocrinol Metab. 2007;92:925-31.

6. August GP, Julius JR, Blethen SL. Adult height in children with growth hormone deficiency who are treated with biosynthetic growth hormone: the National Cooperative Growth Study experience. Pediatrics. 1998;102:512-6.

7. Blethen SL, Baptista J, Kuntze J, Foley T, LaFranchi S, Johanson $\mathrm{A}$. Adult height in growth hormone $(\mathrm{GH})$-deficient children treated with biosynthetic GH. The Genentech Growth Study Group. J Clin Endocrinol Metab. 1997;82:418-20.

8. Carel JC, Ecosse E, Nicolino M, Tauber M, Leger J, Cabrol S, et al. Adult height after long term treatment with recombinant growth hormone for idiopathic isolated growth hormone deficiency: observational follow up study of the French population based registry. BMJ. 2002;325:70.
9. Cutfield W, Lindberg A, Albertsson-Wikland K, Chatelain P, Ranke MB, Wilton P. Final height in idiopathic growth hormone deficiency: the KIGS experience. KIGS International Board. Acta Paediatr Suppl. 1999;88:72-5.

10. Ranke MB, Lindberg A, Chatelain P, Wilton P, Cutfield W, Albertsson-Wikland K, et al. Derivation and validation of a mathematical model for predicting the response to exogenous recombinant human growth hormone $(\mathrm{GH})$ in prepubertal children with idiopathic GH deficiency. KIGS International Board. Kabi Pharmacia International Growth Study. J Clin Endocrinol Metab. 1999;84:1174-83.

11. Ranke MB, Lindberg A, Chatelain P, Wilton P, Cutfield W, AIbertsson-Wikland $\mathrm{K}$, et al. Predicting the response to recombinant human growth hormone in Turner syndrome: KIGS models. KIGS International Board. Kabi International Growth Study. Acta Paediatr Suppl. 1999;88:122-5.

12. Ranke MB, Lindberg A, Chatelain P, Wilton P, Cutfield W, AIbertsson-Wikland $K$, et al. Prediction of long-term response to recombinant human growth hormone in Turner syndrome: development and validation of mathematical models. KIGS International Board. Kabi International Growth Study. J Clin Endocrinol Metab. 2000;85:4212-8.

13. Ranke MB, Lindberg A, Ferrandez Longas A, Darendeliler F, Albertsson-Wikland $K$, Dunger $D$, et al. Major determinants of height development in Turner syndrome (TS) patients treated with GH: analysis of 987 patients from KIGS. Pediatr Res. 2007;61:105-10.

14. Ranke MB, Lindberg A, Price DA, Darendeliler F, AlbertssonWikland $\mathrm{K}$, Wilton $\mathrm{P}$, et al. Age at growth hormone therapy start and first-year responsiveness to growth hormone are major determinants of height outcome in idiopathic short stature. Horm Res. 2007;68:53-62.

15. Soriano-Guillen L, Coste J, Ecosse E, Leger J, Tauber M, Cabrol $\mathrm{S}$, et al. Adult height and pubertal growth in Turner syndrome after treatment with recombinant growth hormone. J Clin Endocrinol Metab. 2005;90:5197-204.

16. Wikland KA, Kristrom B, Rosberg S, Svensson B, Nierop AF. Validated multivariate models predicting the growth response to $\mathrm{GH}$ treatment in individual short children with a broad range in GH secretion capacities. Pediatr Res. 2000;48:475-84.

17. Wit JM, Kamp GA, Rikken B. Spontaneous growth and response to growth hormone treatment in children with growth hormone deficiency and idiopathic short stature. Pediatr Res. 1996;39:295-302.

18. Ranke MB, Cutfield WS, Lindberg A, Cowell CT, AlbertssonWikland K, Reiter EO, et al. A growth prediction model for short children born small for gestational age. J Pediatr Endocrinol Metab. 2002;15 Suppl 5:1273.

19. Ranke MB, Lindberg A, Cowell CT, Wikland KA, Reiter EO, Wilton $\mathrm{P}$, et al. Prediction of response to growth hormone treatment in short children born small for gestational age: analysis of data from KIGS (Pharmacia International Growth Database). J Clin Endocrinol Metab. 2003;88:125-31.

20. Ranke MB, Lindberg A, Albertsson-Wikland K, Wilton P, Price $D A$, Reiter EO. Increased response, but lower responsiveness, to growth hormone $(\mathrm{GH})$ in very young children (aged 0-3 years) with idiopathic GH deficiency: analysis of data from KIGS. J Clin Endocrinol Metab. 2005;90:1966-71.

21. Ranke MB, Lindberg $A$, Martin DD, Bakker B, Wilton P, Albertsson-Wikland $K$, et al. The mathematical model for total pubertal growth in idiopathic growth hormone $(\mathrm{GH})$ deficiency suggests a moderate role of $\mathrm{GH}$ dose. J Clin Endocrinol Metab. $2003 ; 88: 4748-53$. 
22. Blethen SL, Compton P, Lippe BM, Rosenfeld RG, August GP, Johanson A. Factors predicting the response to growth hormone $(\mathrm{GH})$ therapy in prepubertal children with GH deficiency. J Clin Endocrinol Metab. 1993;76:574-9.

23. Cole TJ, Hindmarsh PC, Dunger DB. Growth hormone (GH) provocation tests and the response to $\mathrm{GH}$ treatment in $\mathrm{GH}$ deficiency. Arch Dis Child. 2004;89:1024-7.

24. Schonau E, Westermann F, Rauch F, Stabrey A, Wassmer G, Keller $E$, et al. A new and accurate prediction model for growth response to growth hormone treatment in children with growth hormone deficiency. Eur J Endocrinol. 2001;144:13-20.

25. Reiter EO, Price DA, Wilton P, Albertsson-Wikland K, Ranke MB. Effect of growth hormone $(\mathrm{GH})$ treatment on the near-final height of 1258 patients with idiopathic $\mathrm{GH}$ deficiency: analysis of a large international database. J Clin Endocrinol Metab. 2006;91:2047-54.

26. Hibi I, Tanaka T. Final height of patients with idiopathic growth hormone deficiency after long-term growth hormone treatment. Committee for Treatment of Growth Hormone Deficient Children, Growth Science Foundation, Japan. Acta Endocrinol (Copenh). 1989;120:409-15.

27. Wit JM, Faber JA, van den Brande JL. Growth response to human growth hormone treatment in children with partial and total growth hormone deficiency. Acta Paediatr Scand. 1986;75:767-73.

28. Frasier SD, Costin G, Lippe BM, Aceto T Jr., Bunger PF. A doseresponse curve for human growth hormone. J Clin Endocrinol Metab. 1981;53:1213-7.

29. Preece MA, Tanner JM, Whitehouse RH, Cameron N. Dose dependence of growth response to human growth hormone in growth hormone deficiency. J Clin Endocrinol Metab. 1976;42:477-83.

30. Birnbacher R, Riedl S, Frisch H. Long-term treatment in children with hypopituitarism: pubertal development and final height. Horm Res. 1998;49:80-5.

31. Albertsson-Wikland $\mathrm{K}$. The effect of human growth hormone injection frequency on linear growth rate. Acta Paediatr Scand. 1987;337:110-6.

32. Frisch $\mathrm{H}$, Birnbacher R. Final height and pubertal development in children with growth hormone deficiency after long-term treatment. Horm Res. 1995;43:132-4.

33. Bright GM, Julius JR, Lima J, Blethen SL. Growth hormone stimulation test results as predictors of recombinant human growth hormone treatment outcomes: preliminary analysis of the national cooperative growth study database. Pediatrics. 1999;104:1028-31.

34. Radetti G, Buzi F, Cassar W, Paganini C, Stacul E, Maghnie M. Growth hormone secretory pattern and response to treatment in children with short stature followed to adult height. Clin Endocrinol (Oxf). 2003;59:27-33.

35. Guyda HJ. Four decades of growth hormone therapy for short children: what have we achieved? J Clin Endocrinol Metab. 1999;84:4307-16.

36. Rosenbloom AL. A mathematical model for predicting growth response to growth hormone replacement therapy--a useful clinical tool or an intellectual exercise? J Clin Endocrinol Metab. 1999;84:1172-3.

37. De Boer $H$, van der Veen EA. Why retest young adults with childhood-onset growth hormone deficiency? J Clin Endocrinol Metab. 1997;82:2032-6.

38. Longobardi S, Merola B, Pivonello R, Di Rella F, Di Somma C, Colao A, et al. Reevaluation of growth hormone $(\mathrm{GH})$ secretion in 69 adults diagnosed as $\mathrm{GH}$-deficient patients during childhood. J Clin Endocrinol Metab. 1996;81:1244-7.

39. Tillmann V, Buckler JM, Kibirige MS, Price DA, Shalet SM, Wales JK, et al. Biochemical tests in the diagnosis of childhood growth hormone deficiency. J Clin Endocrinol Metab. 1997;82:531-5.

40. Santos C, Essioux L, Teinturier C, Tauber M, Goffin V, Bougneres $\mathrm{P}$. A common polymorphism of the growth hormone receptor is associated with increased responsiveness to growth hormone. Nat Genet. 2004;36:720-4.

41. Jorge AA, Marchisotti FG, Montenegro LR, Carvalho LR, Mendonça BB, Arnhold IJ. Growth hormone (GH) pharmacogenetics: influence of $\mathrm{GH}$ receptor exon 3 retention or deletion on first-year growth response and final height in patients with severe GH deficiency. J Clin Endocrinol Metab. 2006;91:1076-80.

\section{Endereço para correspondência:}

Ivo J. P. Arnhold

Av. Dr. Enéas de Carvalho Aguiar, 155, PAMB, $2^{\circ}$ andar, bloco 6 05403-900 São Paulo, SP

E-mail: iarnhold@usp.br. 